\title{
Regulation of iron-uptake systems in Vibrio vulnificus, a ferrophilic bacterium
}

\author{
Sung-Heui Shin \\ Department of Microbiology, College of Medicine, Chosun University, Gwangju, Korea
}

Received March 22, 2021

Revised April 14, 2021

Accepted May 4, 2021

Corresponding author

Sung-Heui Shin

Department of Microbiology,

College of Medicine, Chosun

University, 309 Pilmin-daero,

Dong-gu, Gwangju 61452, Korea

Tel: +82-62-230-6352

Fax: +82-62-608-5314

E-mail: shsin@chosun.ac.kr

ORCID:

https://orcid.org/0000-0003-0688-4161

\begin{abstract}
Vibrio vulnificus is a gram-negative ferrophilic bacterium that causes necrotizing wound infections and fatal septicemia, which mainly occur in patients with elevated levels of iron in serum or tissue, despite the presence of well-developed bacterial multiple iron-uptake systems (IUSs). These IUSs play important roles in the pathogenesis of $V$. vulnificus infections and are primarily regulated at the transcriptional level by a ferric uptake regulator called Fur responding to iron availability and their own specific regulators. Recent studies have shown that the IUSs are also controlled by other global regulators, including cyclic AMP-receptor protein responding to carbon availability and $\mathrm{SmcR}$, a master regulator of the quorum-sensing system responding to bacterial density. This review presents an update on this sophisticated regulation of IUSs in V. vulnificus.
\end{abstract}

Keywords: Vibrio vulnificus; Iron; Quorum sensing; Ferric uptake regulator; cAMP-receptor protein

\section{INTRODUCTION}

Iron $(\mathrm{Fe})$ is the fourth most abundant metal on Earth and is an essential ingredient for all living things, including bacteria. However, bacteria must be able to acquire Fe to settle, proliferate, and cause infections in their hosts, and therefore, many pathogenic bacteria have their own well-developed iron-uptake systems (IUSs). The activity of IUSs has long been recognized as being closely related to the virulence of pathogenic bacteria. Therefore, IUSs have been considered a target in the development of vaccines or new therapeutic modalities [1-3].

Fe is quickly oxidized to an insoluble form in an environment where oxygen is present at neutral $\mathrm{pH}$. Therefore, the concentration of soluble Fe that is easily available to most living things is very low. The human body contains $3-5 \mathrm{~g}$ of $\mathrm{Fe}$, most of which is intracellular. The majority of Fe in the body is present as hemoglobin in erythrocytes. Extracellular $\mathrm{Fe}$ is rapidly removed by high-affinity Fe-binding proteins, such as transferrin, ferritin, and lactoferrin [1-3]. Thus, a normal human body contains a very low concentration of free $\mathrm{Fe}$ and is an Fe-deficient environment. This low Fe-availability is considered an innate defense mechanism called "innate nutritional immunity", which helps in suppressing the growth of pathogenic bacteria [2]. However, the concentration of easily available Fe as well as the degree of Fe saturation of transferrin or lactoferrin can be increased, thereby allowing pathogenic bacteria to grow in many pathological conditions, such as liver diseases, hemosiderosis, as well as during transfusions and chemotherapy [1-3]. 
Bacteria have adapted to Fe-deficient environments, developing their own unique IUSs that can efficiently absorb environmental Fe present at low concentrations. Generally, bacterial IUSs can be divided into three types. First, several bacterial species can obtain Fe by expressing specific receptors that directly bind to Fe-withholding proteins such as hemoglobin, transferrin, and lactoferrin [4]. For instance, Neisseria and Haemophilus species express receptors that are unique only to human transferrin and lactoferrin. Other bacterial species can acquire Fe by directly binding to heme proteins such as hemin and hemoglobin by expressing receptors specific to heme [5-7]. Second, most bacteria, except Neisseria or Haemophilus, can produce small molecules $(600-1,500 \mathrm{kDa})$, called siderophores, with a high affinity for Fe. Most siderophores have a higher affinity for Fe than Fe-withholding proteins such as transferrin or lactoferrin so that they can deprive these proteins of Fe. The siderophore-Fe complexes are again absorbed through specific receptors. Many bacteria produce one or more siderophores [8,9]. Third, some bacteria can also use xenosiderophores (also called heterologous or exogenous siderophores) produced by other bacteria or fungi [10-15] through the phenomenon called "siderophore piracy". This siderophore piracy may play an important role in the survival and proliferation of pathogenic bacteria especially in mixed bacterial environments.

Fe is an essential substance for efficient energy production in bacterial cells. Therefore, the amount of Fe required by bacteria is closely related to the metabolic activity in bacterial cells. The more active the metabolism, the more Fe is required to support efficient energy production. However, excessive Fe can be toxic because this can promote free radical formation within bacterial cells. Therefore, the expression of IUSs in bacterial cells must be thoroughly and sensitively controlled [16].

\section{A FERROPHILIC BACTERIUM Vibrio vulnificus}

Vibrio vulnificus is an opportunistic pathogen that can cause necrotizing fasciitis or fatal sepsis mainly in patients with underlying diseases such as alcoholic hepatitis, cirrhosis, and hemosiderosis [17-20]. Most of these patients have elevated serum Fe concentrations [21,22]. Fe has long been known to play an important role in the pathogenesis of $V$. vulnificus infections [23]. First, an elevated Fe concentration is directly related to host sensitivity to $V$. vulnificus [21,22]. In a mouse experimental model, the $50 \%$ lethal dose was significantly reduced by exogenous Fe administration [24, 25]. These Fe-overloaded mice are used as the most susceptible experimental animal for $V$. vulnificus infection. Second, an elevated Fe concentration promotes the production of exotoxins from $V$. vulnificus, and the production of hemolysin and metalloproteinase is increased in response to iron concentration [26,27]. Third, $V$. vulnificus is a ferrophilic or Fe-sensitive bacterium that requires higher levels of soluble Fe for growth than other pathogenic bacteria do [28,29]. The reason for this ferrophilic characteristic is the low activity of $V$. vulnificus well-equipped and versatile IUSs [30]. For example, although $V$. vulnificus produces several siderophores, the amount produced is very low compared to those produced by other pathogenic bacteria.

\section{IUSs IN V. vulnificus}

\section{Vulnibactin-mediated IUS}

$V$. vulnificus produces a siderophore called vulnibactin that has a very high affinity for Fe and is structurally classified as catechol or phenolate siderophore [31,32]. Virulent $V$. vulnificus strains generally produce vulnibactin and use $\mathrm{Fe}$ bound to transferrin, but less virulent or avirulent $V$. vulnificus strains do not produce vulnibactin and are unable to use transferrin-bound Fe [33]. Besides vulnibactin, other siderophores are known to be produced, but vulnibactin has the greatest effect on the growth of $V$. vulnificus in Fe-deficient environments [34].

Genes involved in the production of vulnibactin were first discovered in the ven operon [35]. The venB gene shows $41 \%$ homology at the amino acid level with and the Escherichia coli ent $B$ gene, which encodes isochorismatase, and this enzyme is required for the synthesis of a precursor (2,3-dihydroxybenzoic acid) of enterobactin, a catechol siderophore produced by $E$. coli. $V$. vulnificus containing a mutation in ven $B$ can neither produce vulnibactin nor use Fe bound to transferrin, resulting in the weakened virulence. The vis gene encoding vulnibactin-specific isochorismate synthase is also involved in the synthesis of vulnibactin as a mutation in this gene prevents the production of vulnibactin 
and the use of $\mathrm{Fe}$ bound to transferrin [36,37]. In addition, vvs $A B$, which encodes a member of the nonribosomal peptide synthase, is also required for the biosynthesis of vulnibactin, and a mutation in either $v v s A$ or $v v s B$ decreases the production of vulnibactin in $V$. vulnificus [38]. These show that various enzymes are required for the synthesis of vulnibactin and that vulnibactin has a higher affinity for Fe than transferrin. Thus, $V$. vulnificus can use Fe bound to transferrin through vulnibactin.

Extracellular vulnibactin binds Fe to form the Fe-vulnibactin complex, which is then absorbed via its cognate specific receptor, VuuA, located on the outer membrane of $V$. vulnificus. The $72 \mathrm{kDa}$ VuuA receptor is encoded by vuuA, and a mutation in vuuA prevents the internalization of Fe-vulnibactin into the cytoplasm and inhibits the ability of $V$. vulnificus to use Fe bound to transferrin to grow [39].

\section{Hydroxamate siderophore-mediated IUS}

Virulent $V$. vulnificus strains produce both vulnibactin and a hydroxamate siderophore, but avirulent $V$. vulnificus strains only produce the hydroxamate siderophore [31]. The hydroxamate siderophore can also promote the proliferation of $V$. vulnificus in Fe-deficient environments [31,34]. However, neither the structure of this siderophore nor the genes related to hydroxamate siderophore-mediated IUS have been described.

\section{Xenosiderophore-mediated IUSs}

Desferrioxamine is a hydroxylate siderophore produced by Streptomyces pilosus, and it has long been used as an Fe-chelating agent for the treatment of Fe overload [40]. Several pathogenic bacteria, including $V$. vulnificus, can absorb Fe using this xenosiderophore [10-15], and this has been a major limitation in the use of desferrioxamine as a therapeutic Fe-chelating agent. As a result, new Fe-chelating agents that inhibit the growth of pathogenic bacteria capable of utilizing desferrioxamine, including $V$. vulnificus, have been developed [28,41-44]. Accordingly, these Fe-chelating agents have the potential to prevent and treat fatal infections caused by such pathogenic bacteria [45].

In $V$. vulnificus, desferrioxamine is utilized via its cognate receptor called DesA, which is an Fe-responsive outer membrane protein of $78 \mathrm{kDa}[11,12]$. A mutation in desA abolishes the ability of $V$. vulnificus to use desferrioxamine and inhibits the growth of $V$. vulnificus in the presence of desferrioxamine. This DesA-mediated IUS is widespread in clinical or environmental isolates of $V$. vulnificus [15].

$V$. vulnificus can also use another xenosiderophore called aerobactin, which is produced by $E$. coli, via the cognate receptor lutA, an outer membrane protein of $76 \mathrm{kDa}$ [13]. A mutation in iutA abolishes the ability of $V$. vulnificus to utilize aerobactin for Fe acquisition and can inhibit the growth of $V$. vulnificus in the presence of aerobactin.

$\mathrm{Fe}$-aerobactin or Fe-desferrioxamine is transported into the cytoplasm by an ATP-binding cassette $(\mathrm{ABC})$ transport system containing VatB, VatD, and VatB. The vatC, vatD, and $v a t B$ genes encode an ATP-binding protein, a periplasmic binding protein, and an inner membrane permease, respectively. A mutation in these genes also results in the loss of the ability of $V$. vulnificus to utilize aerobactin as well as desferrioxamine [13]. This indicates that this $\mathrm{ABC}$ transport system is shared between the IutA-mediated and DesA-mediated IUSs.

\section{Heme receptor-mediated IUS}

The first identified heme receptor was a $77 \mathrm{kDa}$ outer membrane protein called HupA, which responds to Fe availability [6]. A mutation in hupA encoding this receptor protein prevents $V$. vulnificus from using hemin or hemoglobin as an Fe source and reduces virulence in mice and in tissue culture $[46,47]$. This implies that HupA plays a significant role in the pathogenesis of $V$. vulnificus infections. Recently, a new heme receptor called HvtA $(79 \mathrm{kDa})$ was identified, and this receptor acts with HupA to enable $V$. vulnificus to make more effective use of hemin or hemoglobin [48]. The growth of $V$. vulnificus is not solely impaired by a mutation in hvtA. Accordingly, HvtA, in conjunction with HupA, is needed for optimal hemin utilization, and HupA and HvtA are considered major and minor heme receptors, respectively. In addition, the HupBCD components of the $A B C$ transport system are shared by HupA and HvtA. Therefore, a mutation in the hupBCD genes also abolishes the ability of $V$. vulnificus to utilize hemin or hemoglobin in the absence of vulnibactin [49]. 


\section{REGULATION OF IUSs BY Fur AND THEIR SPECIFIC REGULATORS}

\author{
Regulation of the vulnibactin-mediated IUS by \\ Fur (Figs. 1 and 2)
}

The expression of bacterial IUSs is controlled by a global regulator called ferric uptake regulator (Fur), which is primarily responsive to Fe availability [50]. In Fe-sufficient environments, the Fe-Fur complex binds to a specific sequence called the "Fur box" in the regulatory region of its target genes [51]. This prevents the binding of RNA polymerase and represses the transcription of target genes. By contrast, under Fe-deficient environments, apo-Fur dissoci-

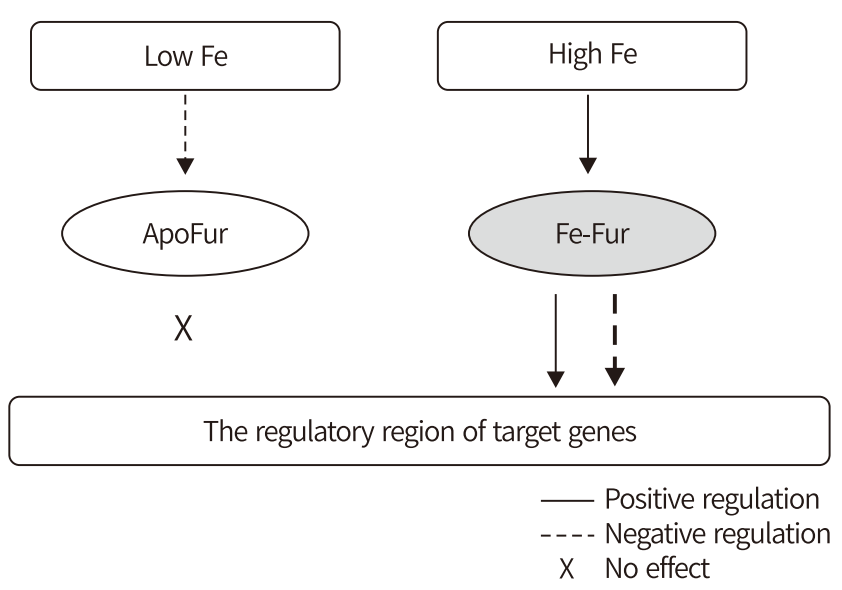

Fig. 1. Schematic illustration of the regulation of target genes by $\mathrm{Fe}$ and ferric uptake regulator (Fur) in Vibrio vulnificus. The thickness of line indicates the main function of Fur in $V$. vulnificus. ates from the Fur box so that the transcription of the target genes is derepressed.

The expression of the vulnibactin-mediated IUS is primarily controlled by Fur. A Fur box is not present in the upstream region of ven $B$ which is transcribed in a polycistronic operon [35]. The presence of a Fur box in the regulatory region of vis remains to be experimentally determined. However, the expression of both ven $B$ and vis is negatively regulated by Fe or derepressed by a mutation in fur $[35,52]$. A Fur box is present at the regulatory regions of $v v S A B$ and vuuA, and the expression of these genes is also negatively regulated by Fe and derepressed by a mutation in fur $[38,39$, 52-55]. Thus, the transcription of genes involved in the vulnibactin-mediated IUS is primarily controlled by Fur, a transcription repressor responding to Fe availability.

\section{Regulation of the heme receptor-mediated IUS by Fur and HupR (Figs. 1 and 3 )}

The expression of hupA, hvtA, and hupBCD is negatively regulated by Fe. A Fur box is present in the regulatory region of these genes, and a mutation in fur de-represses the expression of these genes [46-49]. This indicates that Fe is a primary essential signal for the expression of the heme receptor-mediated IUS. In addition, HupR, a LysR-like transcription activator, acts as a transcription activator for the expression of hupA only when hemin or hemoglobin is supplied as an Fe source in Fe-deficient conditions [56]. The expression of hupA occurs at relatively low levels even in the
A

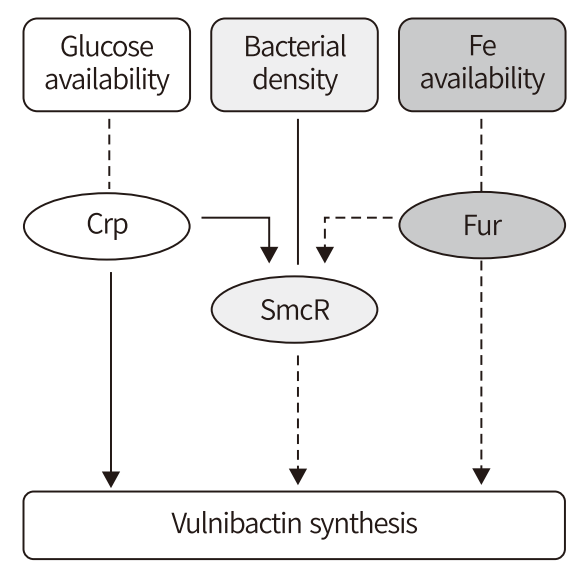

B

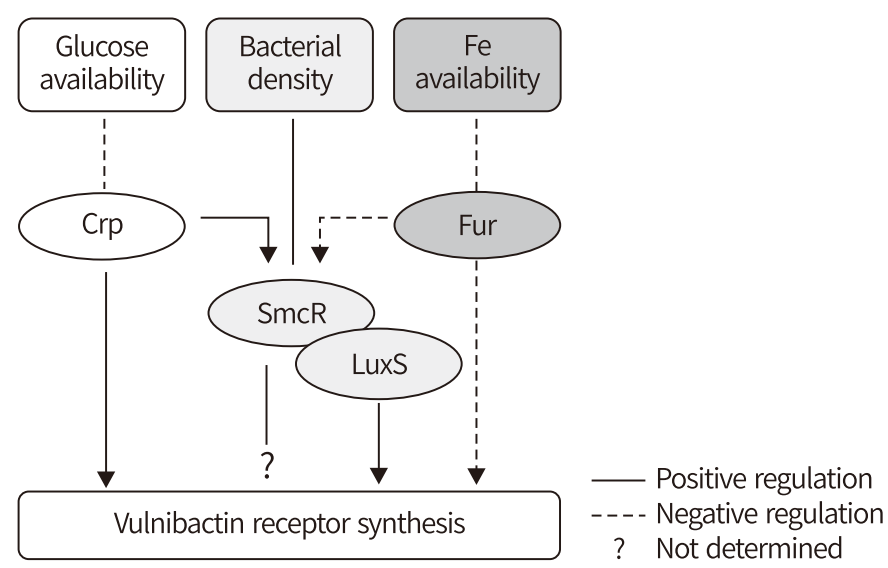

Fig. 2. Schematic illustration of the regulation of vulnibactin (A) and vulnibactin receptor protein (B) production in Vibrio vulnificus. Several enzymes such as vis, ven $B$, and wsAB are known to be involved in vulnibactin synthesis. Crp: cyclic AMP-receptor protein, SmcR: a master regulator of the autoinducer-2mediated quorum sensing system, LuxS: an enzyme required for autoinducer-2 synthesis, Fur: ferric uptake regulator. 
absence of hemin or hemoglobin or the absence of HupR. A mutation in hupR decreases the expression of hupA only in the presence of hemin or hemoglobin as an Fe source. HupR upregulates the expression of hupA but not that of $h v t A$ in the presence of hemin or hemoglobin. It is hypothesized that a small amount of heme can be transported to the cytoplasm via HvtA, resulting in the upregulation of hupA
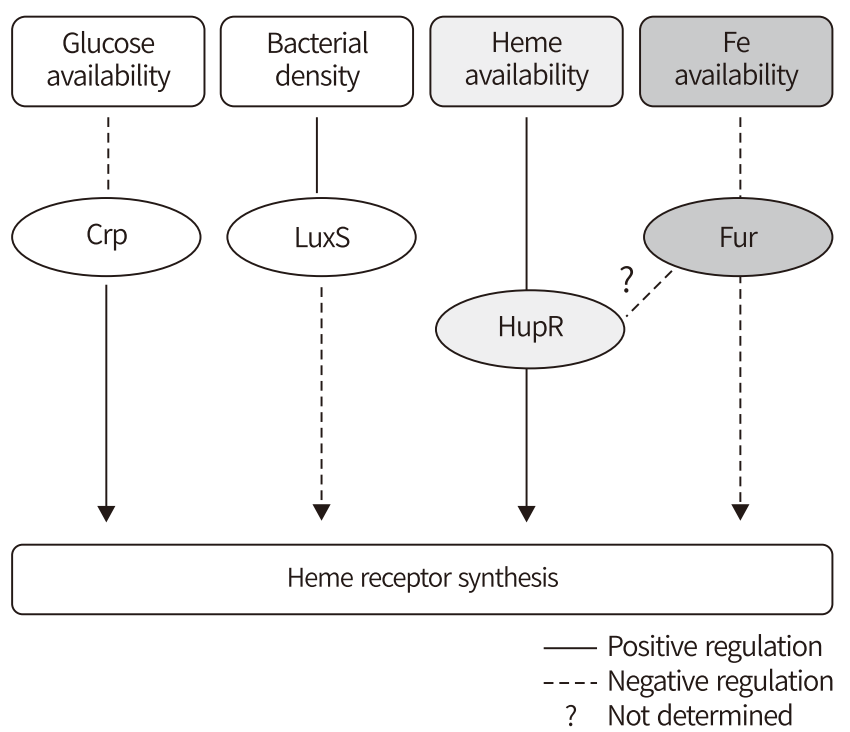

Fig. 3. Schematic illustration of the regulation of heme receptor protein production in Vibrio vulnificus. HupR acts as a transcription activator only in the presence of heme. Other abbreviations are the same as those in Fig. 2.

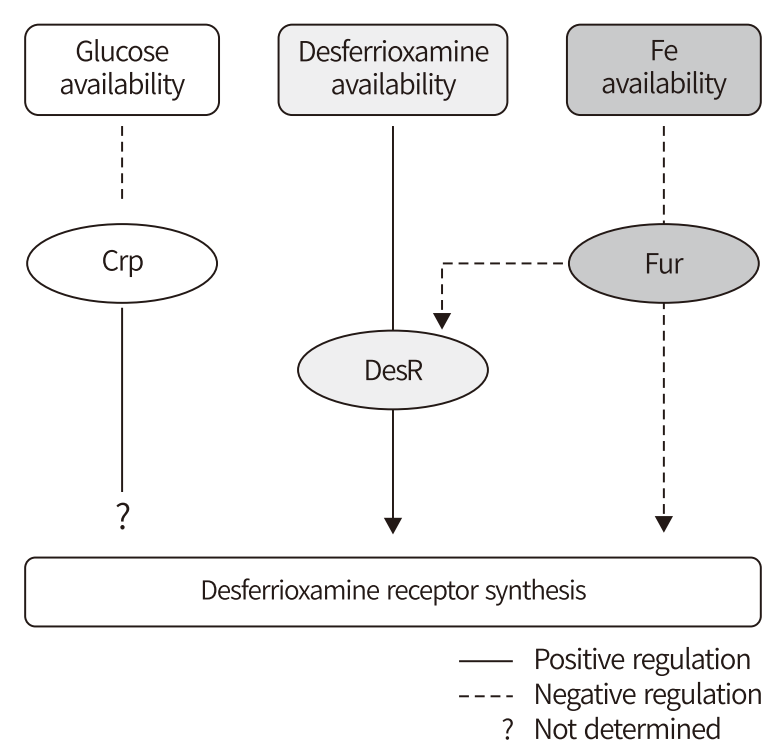

Fig. 4. Schematic illustration of the regulation of desferrioxamine receptor protein production in Vibrio vulnificus. DesR acts as a transcription activator only in the presence of desferrioxamine. Other abbreviations are the same as those in Fig. 2. transcription by HupR [49]. Whether HupR itself is Fe-responsive remains unproven. Thus, HupR is a local or specific, but not essential, regulator that controls the expression of hupA in response to the presence of heme.

\section{Regulation of the DesA-mediated IUS by Fur and DesR (Figs. 1 and 4)}

In Fe-deficient environments, desA is expressed only in the presence of desferrioxamine. DesR, an AraC-like transcription activator, is essentially required for the expression of $\operatorname{des} A[11,12]$. Putative Fur boxes are present in the regulatory regions of both des $A$ and desR, indicating that $\mathrm{Fe}$ is a primary signal for their expression. A mutation in des $R$ results in a loss of production of DesA even in Fe-deficient environments or in the presence of desferrioxamine, which indicates that Fe-responsive DesR is a local, specific, and essential activator responding to the presence of desferrioxamine.

\section{Regulation of the lutA-mediated IUS by Fur and lutR (Figs. 1 and 5)}

The expression of iutA is also under the control of Fur $[13,57]$, which indicates that Fe is a primary signal for the expression of iutA. IutR, a GntR-like transcription repressor, is also involved in the expression of iutA [13]. IutR itself is

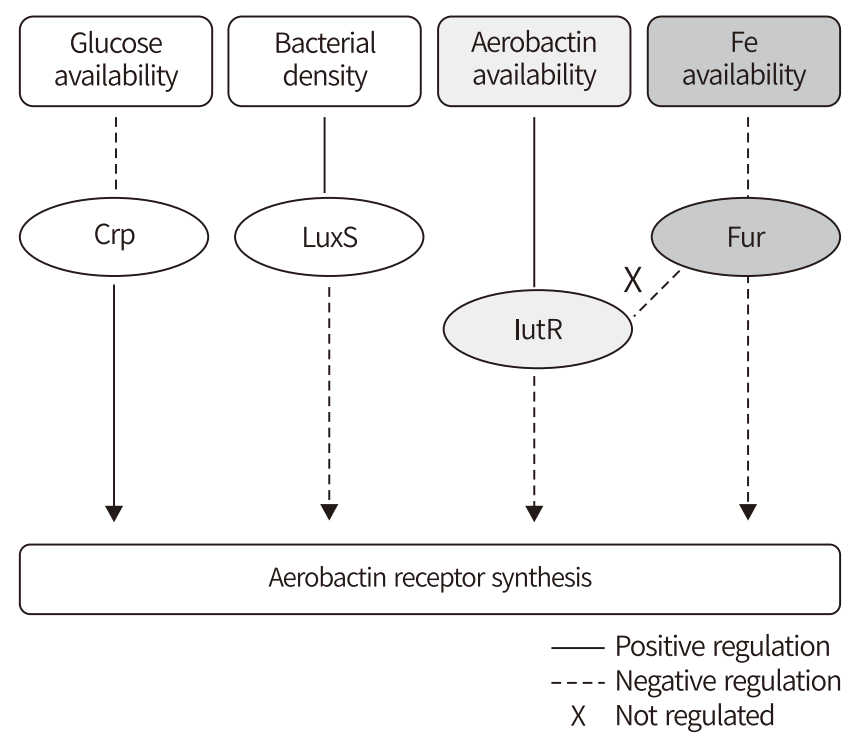

Fig. 5. Schematic illustration of the regulation of aerobactin receptor protein production in Vibrio vulnificus. lutR acts as a transcription repressor in the absence of exogenous aerobactin, whereas in the presence of aerobactin, the lutR-aerobactin complex (probable but not proved) derepresses lutA production. Other abbreviations are the same as those in Fig. 2. 
not regulated by Fe. The expression of iutA occurs at low levels even in the absence of exogenous aerobactin [13,57]. When aerobactin is supplied exogenously, iutA is fully expressed. A mutation in iutR derepresses the expression of iutA regardless of the presence of aerobactin. IutR binds directly to the regulatory region of iutA in the absence of aerobactin, thereby repressing the expression of iutA, whereas in the presence of aerobactin, iutA expression is derepressed by the interaction of IutR with an inducer (probably Fe-aerobactin), leading to a loss of the DNA binding affinity [13]. Accordingly, IutR acts as a local and specific repressor responding to the presence of aerobactin in iron-deficient environments. The expression of vat $C$, vat $D$, and vat $B$ encoding components of the $\mathrm{ABC}$ transport system is also under the negative control of Fe-Fur [13].

\section{REGULATION OF IUSs BY cAMP OR Crp}

The expression of virulence factors is under the control of several global regulators in pathogenic bacteria including $V$. vulnificus [58]. Of these global regulators, cyclic AMP and its receptor protein (Crp) complex are primarily responsive to carbon availability and regulate the expression of a variety of genes, including those related to iron metabolism in $E$. coli [51]. In $V$. vulnificus, cAMP or Crp also regulates the expression of a variety of virulence factors $[59,60]$.

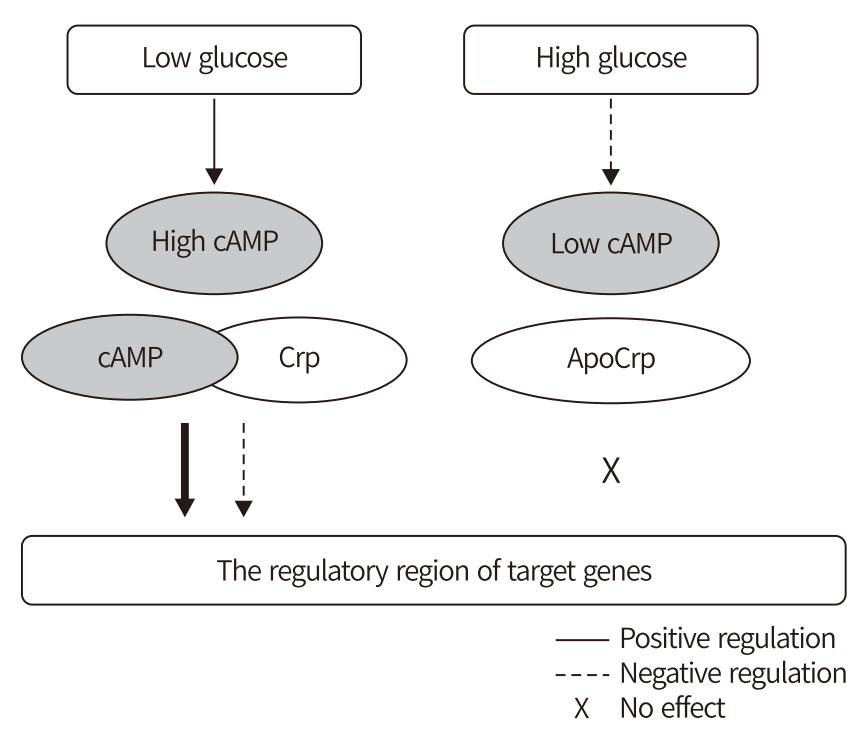

Fig. 6. Schematic illustration of the regulation of target genes by the cyclic AMP (cAMP)-cAMP receptor protein (Crp) complex in Vibrio vulnificus. The thickness of line indicates the main function of $\mathrm{Crp}$ in $V$. vulnificus.
The expression of vis and vuuA is positively regulated by cAMP or Crp (Figs. 2 and 6) [52]. A mutation in cyaA, which encodes adenylate cyclase required for the synthesis of cAMP, or crp, which encodes Crp protein, downregulates the expression of vis and vuuA and attenuates the ability of $V$. vulnificus to use transferrin-bound Fe. The cAMPCrp complex is thought to promote the progression of RNA polymerase by binding to a specific base sequence called the Crp-binding site in the regulatory region of vis or vuuA although direct binding remains to be experimentally determined. The effect of cAMP or Crp on the expression of ven $B$ or vvs $A B$ also remains to be determined.

The expression of hupA is also under the positive control of Crp (Figs. 3 and 6) [46,47]. The cAMP-Crp complex promotes the progression of RNA polymerase by binding to a specific base sequence in the regulatory region of hupA [46]. A mutation in $c y a A$ or $c r p$ decreases the expression of hupA. Moreover, the addition of exogenous cAMP increases the expression of hupA in the cyaA-mutated background, whereas the addition of glucose dose-dependently decreases the expression of hupA [47].

In addition, the expression of iutA is under the positive control of Crp (Figs. 5 and 6) [57]. A mutation in cyaA or crp also decreases the expression of iutA. As with hupA, the expression of iutA in the cyaA-mutated background was increased by the addition of exogenous cAMP and is dose-dependently decreased by the addition of glucose.

The regulation of IUSs by cAMP or Crp indicates that the amount of iron required by $V$. vulnificus is controlled in accordance with the activity of carbon metabolism via the cAMP-Crp complex.

\section{REGULATION OF IUSS BY QUORUM SENSING}

In many pathogenic bacteria including $V$. vulnificus, harmonious organization of virulence gene expression is a key factor in appropriate colonization, invasion, in vivo growth, and in situ toxin production [61]. The cell-to-cell signaling system known as quorum sensing (QS) modulates the expression of virulence factors and synchronizes group behavior for improved survival [62]. In $V$. vulnificus, the autoinducer-2 (AI-2)-mediated QS system has been well studied $[63,64]$ despite the presence of other QS signal mol- 


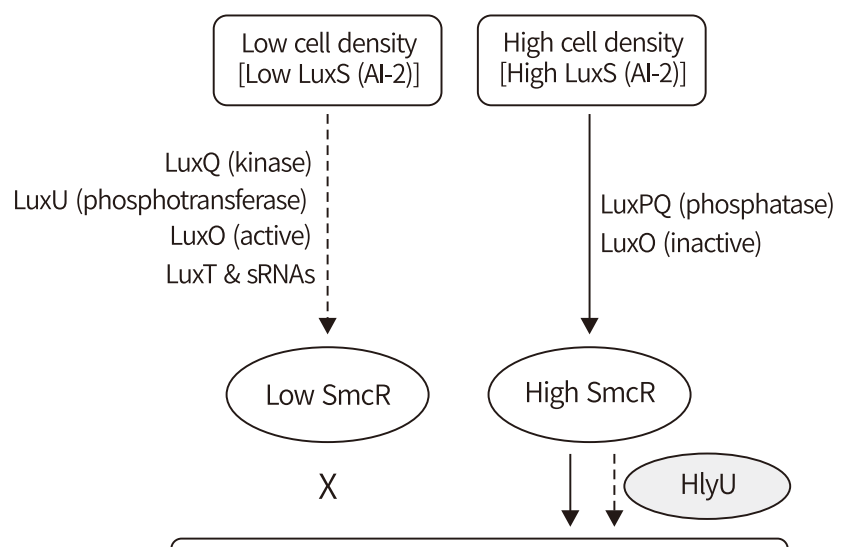

The regulatory region of target genes

- Positive regulation

---- Negative regulation

$X$ No effect

Fig. 7. Schematic illustration of the regulation of target genes by the autoinducer-2 (Al-2)-mediated quorum sensing system in Vibrio vulnificus. HlyU is a small regulatory protein, whose expression is directly and negatively regulated by SmcR, a master regulator of the Al-2mediated quorum sensing system. Details are described in the text.

ecules [65]. V. vulnificus possesses a well-organized QS circuit (Fig. 7), consisting of LuxS (AI-2 synthetase), LuxP-LuxQ (membrane bound sensor proteins), LuxU-LuxO (response regulators), and SmcR (the master transcriptional regulator), all of which are involved in the regulation of virulence factors, such as metalloprotease and hemolysin [65]. Moreover, small RNAs are involved in the repression of SmcR [66]. In addition, LuxT acts as a negative transcriptional regulator of SmcR [66-68].

At low cell densities, the concentration of AI-2 is insufficient to enable QS, LuxQ acts as a kinase and adds a phosphate group to LuxU, which, in turn, transfers the phosphate group to LuxO. LuxO is activated by phosphorylation, and together with RpoN (a nitrogen-limitation sigma factor), sRNAs, and Hfq (a sRNA binding protein), it acts to suppress the production of SmcR. The activated LuxO can also stimulate LuxT, which inhibits the expression of $s m c R$ [68]. By contrast, at high cell densities, $\mathrm{AI}-2$ is present at sufficient concentration to interact with LuxP-LuxQ and modulate their function to a phosphatase, which dephosphorylates LuxO. The dephosphorylated LuxO is inactive and can no longer inhibit SmcR. SmcR can therefore activate or repress the expression of target genes, such as $v p E$ which encodes a metalloprotease and $v$ hA which encodes a hemolysin [68].

A previous study [53] reported that SmcR acts as a tran- scriptional repressor of the expression of $V V S A B$ by directly binding to the regulatory region of $V V S A B$, especially in Fe-deficient conditions (Fig. 2). A mutation in $s m c R$ derepresses vulnibactin production. The binding site of SmcR overlaps with that of Fe-Fur in the regulatory region of $v v s A B$. However, Fe-Fur binds to the regulatory region of $V V S A B$ with higher affinity than SmcR. Therefore, Fur is the key repressor that prevents the overexpression of $V v S A B$ in response to Fe in Fe-sufficient environments, whereas SmcR is another key repressor that prevents the overexpression of ${ }_{V V S} A B$ in response to bacterial density in Fe-deficient environments. In another study [69], a mutation in luxS slightly decreased the expression of vuuA but slightly increased the expression of iutA and hupA in $V$. vulnificus (Figs. 2, 3 and 5). There is a controversy on the effect of AI-2-QS on the expression of IUSs between the two studies; that is to say, a mutation in $s m c R$ derepresses vulnibactin production, whereas a mutation in $l u x S$ decreases vulnibactin receptor production. This controversy remains unexplainable to date. However, it is undeniable that Fe-Fur and QS cooperate to regulate the expression of IUSs in response to Fe availability for the fine-tuning of the intracellular iron level and improved survival of $V$. vulnificus. Further coherent studies need to reveal details on the interaction between IUSs and QS.

\section{REGULATION OF IUSs BY TEMPERATURE}

The effect of other environmental factors affecting the expression of IUSs in addition to Fe availability has not been extensively studied. $V$. vulnificus resides in estuarine environments and can infect humans, and thus, $V$. vulnificus is capable of sensing and responding to diverse environmental changes for either survival or successful establishment of infections in the human body [70,71]. Upon entering the human body, $V$. vulnificus must withstand a temperature change from $25^{\circ} \mathrm{C}$ to $37^{\circ} \mathrm{C}$. The change of temperature modulates the expression of several virulence factors that are required for the bacterial survival and successful infection [67,72]. $V$. vulnificus modulates the expression of hupA by sensing and responding to the change of temperature especially in clinical isolates $[46,48,49]$. In addition, the change of temperature resulted in increased expression of vuuA and crp, and Crp can then act as a transcriptional 
activator in the expression of vuuA [73]. These indicate that a change of temperature is an environmental signal for the regulation of IUSs. Details on the temperature-mediated regulation of IUSs remain to be determined.

\section{EXOTOXINS AFFECTING Fe AVAILABILITY AND THEIR REGULATION}

\section{Regulation of $V$. vulnificus hemolysin and proteases by Fe-Fur (Fig. 8)}

Most of Fe in the human body exists in the form of hemoglobin in erythrocytes. $V$. vulnificus produces several cytotoxins $[19,20,58]$, especially hemolysin, which have a great impact on Fe availability because they can destroy host cells (especially erythrocytes) to release Fe [74]. There is still much doubt concerning the pathogenic role of hemolysin in infections of $V$. vulnificus [75]. However, even if only a very small amount of active hemolysin is produced, which subsequently destroys only a very small amount of erythrocyte, sufficient Fe is made available for use by $V$. vulnificus.

In addition, $V$. vulnificus is hypothesized to utilize the metalloprotease $\mathrm{VvpE}$ activity to assimilate Fe by degrading heme proteins, transferrin and lactoferrin, thereby releasing Fe and increasing its availability [76. However, this hypothesis has not been supported by further studies [36,37,77]. A mutation in $v p E$ did not affect the ability of $V$. vulnificus to produce vulnibactin or to acquire Fe from transferrin or hemoglobin in in Fe-deficient media or in ascitic fluids obtained from liver cirrhosis patients, which is considered an ex vivo experimental system for $V$. vulnificus. By contrast, a mutation in fur derepressed the production of vulnibactin, and facilitated the assimilation of Fe from transferrin, and a mutation in vis abolished the production of vulnibactin and the assimilation of Fe from transferrin under the same conditions. These results show that vulnibactin is essentially required for the assimilation of Fe from transferrin or hemoglobin, and VvpE has no direct effect on the assimilation of Fe from transferrin or hemoglobin via the vulnibactin-mediated IUS under in vitro or ex vivo conditions. Discrepancies among these studies on the roles of $\mathrm{VvpE}$ in the assimilation of Fe from transferrin or hemoglobin may be due to the use of different experimental conditions and methods.

A Fur box exists in the regulatory region of $v h A$ in $V$. vulnificus [78]. The expression of $w h A$ is repressed by Fe and derepressed by a mutation in fur. However, the extracellular secretion of VvhA hemolysin is conversely increased by $\mathrm{Fe}$ [26,79]. VvhA is secreted extracellularly via the type II general secretion system. The extracellular secretion of VvhA is reduced by a mutation in pilD, which encodes PilD, a component of the type II general secretion system. Fe increases the expression of pilD. Thus, Fe represses the expression of whA via Fur at the transcriptional level but increases the extracellular secretion of $V_{v h A}$ via the type II general secretion system. In addition, extracellular VvhA is digested by

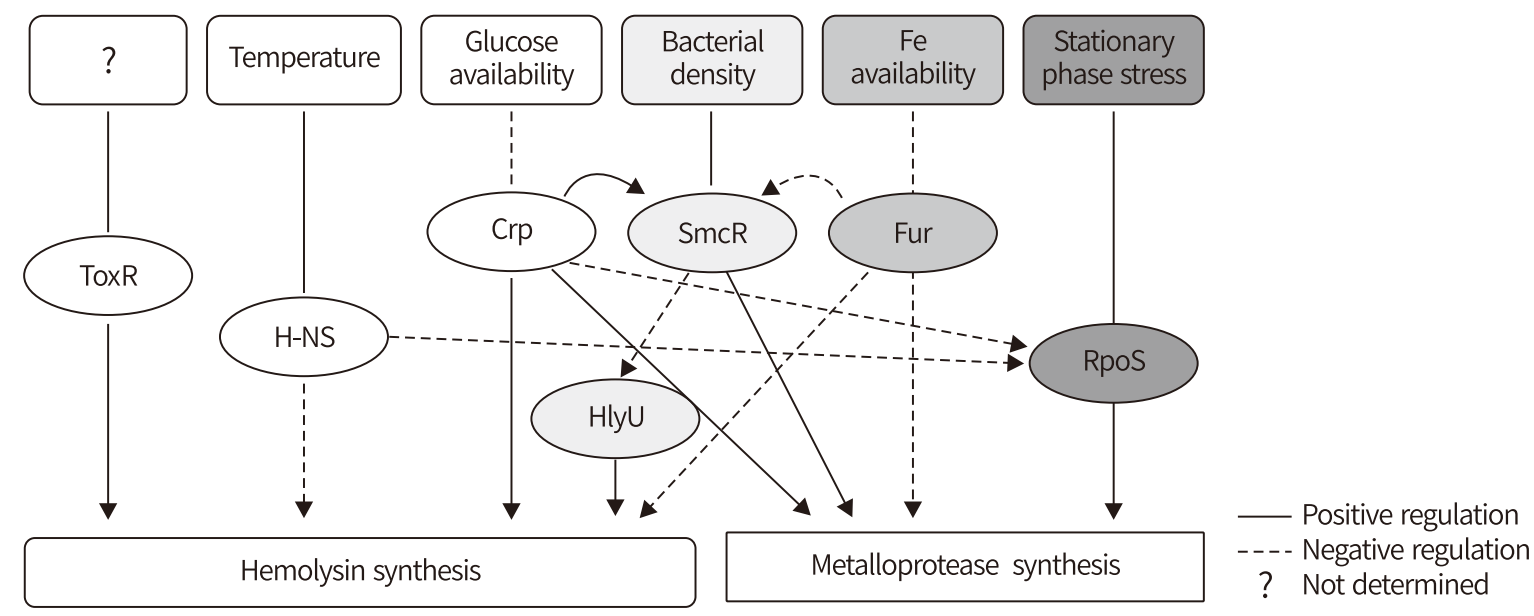

Fig. 8. Schematic illustration of the regulation of hemolysin and metalloprotease production in Vibrio vulnificus. ToxR: a regulator of undefined signal transduction system, which is a homolog of the ToxRS system in Vibrio cholerae. H-NS is a histone-like nucleoid protein, which is involved in the temperature-mediated regulation of gene expression. HlyU is a small regulatory protein, whose expression is directly and negatively regulated by SmcR, a master regulator of the autoinducer-2-mediated quorum sensing system. RpoS is a sigma factor $\left(\sigma^{38}\right)$ for RNA polymerase, which is involved in various stationary stress responses. Other abbreviations are the same as those in Fig. 2. 
VvpE and VvpM, which are produced by $V$. vulnificus. These results indicate that Fur regulates the production of VvhA at the transcriptional level by directly binding to the regulatory region of $w h A$ and at the post-translational level by regulating the expression of two VvhA-degrading exoproteases, namely, VvpE and VvpM.

The expression of $v p D E$, but not $v p M$, is also negatively regulated by direct binding of Fur to its regulatory region [78], indicating that VvpE production is repressed by Fe. However, in other studies [27,36,37,77], Fe increases the expression of $v v p E$ in $V$. vulnificus. Fe stimulated the expression of $v p E$ and the extracellular production of $\mathrm{VvpE}$ even in a crp and/or smcR mutated background. These indicate that Fe increases the expression of $v v p E$ through factor(s) other than Crp and SmcR. This controversy among these studies may also be due to the use of different experimental conditions and methods.

\section{Regulation of hemolysin and protease by cAMP or Crp (Fig. 8)}

The role of the global regulator cAMP-Crp complex in both virulence and general metabolism in $V$. vulnificus has been extensively investigated. cAMP is generated through cleavage of ATP by adenylate cyclase, which is encoded by cyaA in $V$. vulnificus [58]. cAMP acts as a regulator by binding to $\mathrm{Crp}$, which then binds to the regulatory regions of target genes, modulating their expressions. In $V$. vulnificus, several virulence or toxic factors, including $\mathrm{VvpE}$ and $\mathrm{VvhA}$, have been shown to be regulated by the cAMP-Crp system. A mutation in cyaA or crp dramatically decreases VvhA production [59,60]. The cAMP-Crp complex directly binds to the regulatory region of $v W h A$ activating its expression [80,81]. Thus, the cAMP-Crp complex is the primary positive regulator for the expression of $w h A$.

The cAMP-Crp complex also regulates the expression of $w p E$. The complex works in concert with $\mathrm{QS}$ via $\mathrm{SmcR}$ and exerts its effects on the RpoS-mediated expression of ${ } v p E[82,83]$. Two different promoters, namely $\mathrm{L}$ and $\mathrm{S}$, are involved in the expression of $v p E E[31,82,83]$. The activity of the $\mathrm{L}$ promoter is constitutive throughout bacterial growth and is lower than that of the $S$ promotor. The expression of $v p E$ via the $\mathrm{S}$ promotor is induced only in the stationary growth phase, and is dependent on RpoS and positively regulated by both the cAMP-Crp complex and SmcR. The
cAMP-Crp complex and SmcR bind to the different sites of the $S$ promotor. They then act synergistically to activate the expression of $w p E$. In other studies [84-86], a mutation in $c r p$ decreases the expression of $v v p E$ in the early growth stage, whereas a mutation in $s m c R$ decreases the expression of $v v p E$ only at the late growth stage. A double mutation in $c r p$ and $s m c R$ can severely inhibit the expression of $v p E E$ from the early growth stage, and the inhibited expression of $v p E$ is restored only at the early growth stage by a single complementation of $c r p$, but not $s m c R$. Taken together, the cAMP-Crp complex is the primary positive regulator, and SmcR synergistically cooperates with the cAMP-Crp complex for the RpoS-dependent expression of $v v p E$.

In addition, a study reported that $c r p$ and rpoS are expressed more strongly at $26^{\circ} \mathrm{C}$ than at $37^{\circ} \mathrm{C}$ [67]. Other studies $[73,85,86]$ controversially showed that $V$. vulnificus growth is stimulated and $c r p$ or $s m c R$ or $v p E$ is expressed more strongly by a temperature change from $25^{\circ} \mathrm{C}$ to $37^{\circ} \mathrm{C}$. This inconsistency remains to be clarified via further coherent studies.

\section{Regulation of hemolysin and protease by QS}

(Fig. 8)

The QS system has a key role in the coordinate expression of $w h A$ and $v p E$ in $V$. vulnificus. The expression of $v p E E$ is decreased by a mutation in luxS or smcR but increased by a mutation in $\operatorname{luxO}$, whereas the expression of $v$ h $A$ is affected in the opposite manner and increased by a mutation in luxS and smcR but decreased by a mutation of luxO $[63,64,66,67]$. Thus, the QS system in $V$. vulnificus appears to positively regulate the expression of $v p E$, but negatively regulate the expression of $v$ whA.

\section{Regulation of hemolysin and protease by HlyU or HNS (Fig. 8)}

The small regulatory protein $\mathrm{HlyU}$ also regulates the expression of $w$ hhA in $V$. vulnificus . The importance of HlyU in the virulence of this pathogen was first recognized by detection of antibodies against $\mathrm{HlyU}$ in the serum of patients infected by $V$. vulnificus [70]. A mutation in $h l y U$ reduces the cytotoxicity and mouse lethality of $V$. vulnificus and decreases the expression of $w h A$ [70,87]. The expression of whA regulated by HlyU is also dependent on the QS system [88]. SmcR directly binds to a regulatory region of $h l y U$, re- 
pressing the expression of $h l y U$. A double mutation in $s m c R$ and $h l y U$ restores the production of VvhA in the absence of H-NS, a histone-like nucleoid structuring protein that is known to be involved in the temperature-mediated regulation of gene expression [89]. Collectively, SmcR regulates the expression of $w h A$ by repressing $h l y U$.

In addition, a mutation in hns increases the expression of $w h A$ but decreases the expression of $v v p E$ [89]. H-NS directly binds and competes with HlyU for binding to the regulatory region of $w$ hA but not $v v p E$. H-NS positively affects the mRNA level of $r p o S$, and then RpoS positively regulates the expression of $v v p E$. These indicate that the role of $\mathrm{H}-\mathrm{NS}$ is dependent on the QS in the regulation of $w h A$, but not in the regulation of $v p E$.

\section{Regulation of hemolysin and protease by ToxRS (Fig. 8)}

$V$. vulnificus is known to possess another signal transduction system, which is mediated by ToxRS. The ToxRS system of $V$. vulnificus is functionally similar to that of $V$. cholerae. In $V$. cholerae, ToxR regulates the expression of multiple virulence factors including cholera toxin, toxin-coregulated pilus, and accessory colonization factor genes [90]. A mutation in tox $R$ decreases the production of VvhA in $V$. vulnificus [01]. However, there have been no further studies on the ToxRS system in $V$. vulnificus.

\section{INTERACTION AMONG Crp, Fur, QS AND/ OR RpoS}

In addition to the functional cooperation among Crp, Fur, QS and/or RpoS, the direct interaction among these global regulators has been investigated (Fig. 8). Crp primarily responding to carbon availability is involved in the expression of $s m c R$, which encodes the QS master regulator SmcR primarily responding to bacterial density [02]. A mutation in crp impaired $V$. vulnificus growth, decreased AI-2 production, and repressed the expression of $s m c R$. Furthermore, $s m c R$ expression was repressed by glucose in the presence of Crp but not in its absence. A mutation in cyaA also impaired $V$. vulnificus growth and repressed smcR expression. These results indicate that CAMP or CRP modulates the AI2-QS system in response to glucose availability in $V$. vulnificus, demonstrating the presence of a connection between catabolite repression and QS in $V$. vulnificus.

It has not been determined yet whether Crp is involved in the expression of Fur, the global regulator responding to Fe availability, in $V$. vulnificus. However, it has long been known that Crp positively regulates the expression of Fur in E. coli [03]. This suggests that this direct interaction between Crp and Fur may also exist in $V$. vulnificus. Further studies on this interaction are necessary. Interestingly, Fur positively autoregulates the expression of Fur in $V$. vulnificus [94] as well as in E. coli [03]. In $V$. vulnificus, a mutation in fur downregulates the expression of fur in a low-Fe condition, and Fur can directly bind to the regulatory region of fur [94,95].

Fe or Fur also directly or indirectly affects the activity of AI-2-QS system in $V$. vulnificus [66,96].

The expression of $v p E$, a member of the QS regulon, is repressed under iron-rich conditions, and Fe-Fur represses the expression of $s m c R$ by directly and reversibly binding to the regulatory region of $s m c R$. Apo-Fur does not bind to the regulatory region of $s m c R$. Furthermore, Fe-Fur represses the expressions of qrr1-5, which are quorum-regulatory small RNAs that inhibit the expression of $s m c R$ at the posttranscriptional level, by directly binding to their regulatory regions. The expression of $l u x O$ and qrr RNAs is also repressed by Fe independently of Fur. All of these findings indicate that Fe and QS are linked together in the coordinated expression of virulence factors.

Crp also regulates the expression of rpoS [97]. RpoS is an alternative sigma factor $\left(\sigma^{38}\right)$ for RNA polymerase, which is involved in various stationary stress responses. Cellular cAMP levels are inversely correlated with RpoS levels, and a mutation in $c y a A$ or $c r p$ derepresses the expression of $r p o S$. The cAMP-Crp complex represses the expression of $r p o S$ by directly binding to its regulatory region.

\section{CONCLUSION}

$V$. vulnificus is a Gram-negative ferrophilic bacterium that contains well-developed multiple IUSs. $V$. vulnificus causes necrotizing wound infections and fatal septicemia that mainly occur in patients with elevated levels of Fe in serum or tissues. These IUSs play important roles in the pathogenesis of $V$. vulnificus infections, and are sophisticatedly controlled at the transcriptional level by global regulators as well 
as their local or specific regulators. Moreover, functional and direct interactions of global regulators are also present. Accordingly, this sophisticated and delicate regulation of the IUSs allows $V$. vulnificus to sensitively or appropriately respond to a variety of intrinsic or environmental signals to enable bacterial survival and regulation of virulence factors, thereby causing fatal infections. It is expected that a continued understanding of IUSs will contribute to the development of new treatments and preventive measures for $V$. vulnificus infections.

\section{CONFLICT OF INTEREST}

No potential conflict of interest relevant to this article was reported.

\section{REFERENCES}

1. Carver PL. The battle for iron between humans and microbes. Curr Med Chem 2018;25:85-96.

2. Skaar EP, Raffatellu M. Metals in infectious diseases and nutritional immunity. Metallomics 2015;7:926-8.

3. Weinberg ED. Iron and infection. Microbiol Rev 1978;42:4566.

4. Gray-Owen SD, Schryvers AB. Bacterial transferrin and lactoferrin receptors. Trends Microbiol 1996;4:185-91.

5. Mey AR, Payne SM. Haem utilization in Vibrio cholerae involves multiple TonB-dependent haem receptors. Mol Microbiol 2001;42:835-49.

6. Litwin CM, Byrne BL. Cloning and characterization of an outer membrane protein of Vibrio vulnificus required for heme utilization: regulation of expression and determination of the gene sequence. Infect Immun 1998;66:3134-41.

7. Perkins-Balding D, Baer MT, Stojilikovic I. Identification of functionally important regions of a haemoglobin receptor from Neisseria meningitidis. Microbiology (Reading) 2003;149:3423-35.

8. Bagg A, Neilands JB. Molecular mechanism of regulation of siderophore-mediated iron assimilation. Microbiol Rev 1987;51:509-18.

9. Neilands JB. Siderophores. Arch Biochem Biophys 1993;302: $1-3$.

10. Schubert S, Fischer D, Heesemann J. Ferric enterochelin transport in Yersinia enterocolitica: molecular and evolutionary aspects. J Bacteriol 1999;181:6387-95.

11. Aso H, Miyoshi S, Nakao H, Okamoto K, Yamamoto S. Induction of an outer membrane protein of $78 \mathrm{kDa}$ in Vibrio vul- nificus cultured in the presence of desferrioxamine $B$ under iron-limiting conditions. FEMS Microbiol Lett 2002;212:6570.

12. Tanabe $\mathrm{T}$, Takata N, Naka A, Moon YH, Nakao H, Inoue $\mathrm{Y}$, et al. Identification of an AraC-like regulator gene required for induction of the 78-kDa ferrioxamine B receptor in Vibrio vulnificus. FEMS Microbiol Lett 2005;249:309-14.

13. Tanabe T, Naka A, Aso H, Nakao H, Narimatsu S, Inoue $Y$, et al. A novel aerobactin utilization cluster in Vibrio vulnificus with a gene involved in the transcription regulation of the iutA homologue. Microbiol Immunol 2005;49:823-34.

14. Kim CM, Shin SH. Effect of iron-chelator deferiprone on the in vitro growth of staphylococci. J Korean Med Sci 2009; 24:289-95.

15. Kim CM, Park YJ, Shin SH. A widespread deferoxamine-mediated iron-uptake system in Vibrio vulnificus. J Infect Dis 2007;196:1537-45.

16. Andrews SC, Robinson AK, Rodríguez-Quiñones F. Bacterial iron homeostasis. FEMS Microbiol Rev 2003;27:215-37.

17. Park SD, Shon HS, Joh NJ. Vibrio vulnificus septicemia in Korea: clinical and epidemiologic findings in seventy patients. J Am Acad Dermatol 1991;24:397-403.

18. Hlady WG, Klontz KC. The epidemiology of Vibrio infections in Florida, 1981-1993. J Infect Dis 1996;173:1176-83.

19. Strom MS, Paranjpye RN. Epidemiology and pathogenesis of Vibrio vulnificus. Microbes Infect 2000;2:177-88.

20. Baker-Austin C, Oliver JD. Vibrio vulnificus: new insights into a deadly opportunistic pathogen. Environ Microbiol 2018; 20:423-30.

21. Brennt CE, Wright AC, Dutta SK, Morris JG Jr. Growth of Vibrio vulnificus in serum from alcoholics: association with high transferrin iron saturation. J Infect Dis 1991;164:1030-2.

22. Hor LI, Chang TT, Wang ST. Survival of Vibrio vulnificus in whole blood from patients with chronic liver diseases: association with phagocytosis by neutrophils and serum ferritin levels. J Infect Dis 1999;179:275-8.

23. Wright AC, Simpson LM, Oliver JD. Role of iron in the pathogenesis of Vibrio vulnificus infections. Infect Immun 1981; 34:503-7.

24. Hor LI, Chang YK, Chang CC, Lei HY, Ou JT. Mechanism of high susceptibility of iron-overloaded mouse to Vibrio vulnificus infection. Microbiol Immunol 2000;44:871-8.

25. Starks AM, Schoeb TR, Tamplin ML, Parveen S, Doyle TJ, Bomeisl PE, et al. Pathogenesis of infection by clinical and environmental strains of Vibrio vulnificus in iron-dextran-treated mice. Infect Immun 2000;68:5785-93.

26. Kim CM, Shin SH. Iron triggers extracellular secretion of Vibrio vulnificus hemolysin VvhA. Med J Chosun Univ 2010; 34(S):458-64.

27. Kim CM, Kim SC, Shin SH. Iron-mediated regulation of 
metalloprotease VvpE production in Vibrio vulnificus. New Microbiol 2012;35:481-6.

28. Kim CM, Park RY, Choi MH, Sun HY, Shin SH. Ferrophilic characteristics of Vibrio vulnificus and potential usefulness of iron chelation therapy. J Infect Dis 2007;195:90-8.

29. Weinberg ED. Microbial pathogens with impaired ability to acquire host iron. Biometals 2000;13:85-9.

30. Shin SH. Multiple high-affinity iron-uptake systems of Vibrio vulnificus. J Bacteriol Virol 2013;43:168-76.

31. Simpson LM, Oliver JD. Siderophore production by Vibrio vulnificus. Infect Immun 1983;41:644-9.

32. Okujo N, Saito M, Yamamoto S, Yoshida T, Miyoshi S, Shinoda S. Structure of vulnibactin, a new polyamine-containing siderophore from Vibrio vulnificus. Biometals 1994;7:109-16.

33. Stelma GN Jr, Reyes AL, Peeler JT, Johnson CH, Spaulding PL. Virulence characteristics of clinical and environmental isolates of Vibrio vulnificus. Appl Environ Microbiol 1992;58:2776-82.

34. Shin SH, Chung SS, Rhee JH. Phenolate siderophore stimulates growth of Vibrio vulnificus: application of CAS agar diffusion assay-comparison of siderophore production among strains. J Bacteriol Virol 2001;31:325-31.

35. Litwin CM, Rayback TW, Skinner J. Role of catechol siderophore synthesis in Vibrio vulnificus virulence. Infect Immun 1996;64:2834-8.

36. Sun HY, Han SI, Choi MH, Kim SJ, Kim CM, Shin SH. Vibrio vulnificus metalloprotease VvpE has no direct effect on iron-uptake from human hemoglobin. J Microbiol 2006;44:537-47.

37. Kim CM, Park RY, Park JH, Sun HY, Bai YH, Ryu PY, et al. Vibrio vulnificus vulnibactin, but not metalloprotease VvpE, is essentially required for iron-uptake from human holotransferrin. Biol Pharm Bull 2006;29:911-8.

38. Kim IH, Shim JI, Lee KE, Hwang W, Kim IJ, Choi SH, et al. Nonribosomal peptide synthase is responsible for the biosynthesis of siderophore in Vibrio vulnificus MO6-24/O. J Microbiol Biotechnol 2008;18:35-42.

39. Webster AC, Litwin CM. Cloning and characterization of vuuA, a gene encoding the Vibrio vulnificus ferric vulnibactin receptor. Infect Immun 2000;68:526-34.

40. Olivieri NF, Brittenham GM. Iron-chelating therapy and the treatment of thalassemia. Blood 1997;89:739-61.

41. Lesic B, Foulon J, Carniel E. Comparison of the effects of deferiprone versus deferoxamine on growth and virulence of Yersinia enterocolitica. Antimicrob Agents Chemother 2002;46:1741-5.

42. Kim DM, Cho HS, Kang JI, Kim HS, Park CY. Deferasirox plus ciprofloxacin combination therapy after rapid diagnosis of Vibrio vulnificus sepsis using real-time polymerase chain reaction. J Infect 2008;57:489-92.
43. Neupane GP, Kim DM. Comparison of the effects of deferasirox, deferiprone, and deferoxamine on the growth and virulence of Vibrio vulnificus. Transfusion 2009;49:1762-9.

44. Neupane GP, Kim DM. In vitro time-kill activities of ciprofloxacin alone and in combination with the iron chelator deferasirox against Vibrio vulnificus. Eur J Clin Microbiol Infect Dis 2010;29:407-10.

45. Thompson MG, Corey BW, Si Y, Craft DW, Zurawski DV. Antibacterial activities of iron chelators against common nosocomial pathogens. Antimicrob Agents Chemother 2012;56:5419-21.

46. Oh MH, Lee SM, Lee DH, Choi SH. Regulation of the Vibrio vulnificus hupA gene by temperature alteration and cyclic AMP receptor protein and evaluation of its role in virulence. Infect Immun 2009;77:1208-15.

47. Kim SP, Lee GW, Kim CM, Shin SH. Coordinate regulation of Vibrio vulnificus heme receptor HupA expression by cyclic AMP-receptor protein and ferric uptake regulator. J Bacteriol Virol 2012;42:294-304.

48. Datta S, Crosa JH. Identification and characterization of a novel outer membrane protein receptor required for hemin utilization in Vibrio vulnificus. Biometals 2012;25:275-83.

49. Kawano H, Miyamoto K, Yasunobe M, Murata M, Yamahata $\mathrm{E}$, Yamaguchi R, et al. Identification of the heme acquisition system in Vibrio vulnificus M2799. Microb Pathog 2018;117:100-8.

50. Bagg A, Neilands JB. Ferric uptake regulation protein acts as a repressor, employing iron (II) as a cofactor to bind the operator of an iron transport operon in Escherichia coli. Biochemistry 1987;26:5471-7.

51. Zhang Z, Gosset G, Barabote R, Gonzalez CS, Cuevas WA, Saier MH Jr. Functional interactions between the carbon and iron utilization regulators, Crp and Fur, in Escherichia coli. J Bacteriol 2005;187:980-90.

52. Choi MH, Sun HY, Park RY, Kim CM, Bai YH, Kim YR, et al. Effect of the crp mutation on the utilization of transferrin-bound iron by Vibrio vulnificus. FEMS Microbiol Lett 2006;257:285-92.

53. Wen Y, Kim IH, Son JS, Lee BH, Kim KS. Iron and quorum sensing coordinately regulate the expression of vulnibactin biosynthesis in Vibrio vulnificus. J Biol Chem 2012;287:26727-39.

54. Litwin CM, Calderwood SB. Role of iron in regulation of virulence genes. Clin Microbiol Rev 1993;6:137-49.

55. Lee HJ, Lee KH. Identification of the fur-binding site in regulatory region of the vulnibactin-receptor gene in Vibrio vulnificus. J Microbiol Biotechnol 2012;22:46-9.

56. Litwin CM, Quackenbush J. Characterization of a Vibrio vulnificus LysR homologue, HupR, which regulates expression of the haem uptake outer membrane protein, HupA. Microb 
Pathog 2001;31:295-307.

57. Kim CM, Kim SJ, Shin SH. Cyclic AMP-receptor protein activates aerobactin receptor IutA expression in Vibrio vulnificus. J Microbiol 2012;50:320-5.

58. Jones MK, Oliver JD. Vibrio vulnificus: disease and pathogenesis. Infect Immun 2009;77:1723-33.

59. Kim YR, Kim SY, Kim CM, Lee SE, Rhee JH. Essential role of an adenylate cyclase in regulating Vibrio vulnificus virulence. FEMS Microbiol Lett 2005;243:497-503.

60. Kim YR, Lee SE, Kim B, Choy H, Rhee JH. A dual regulatory role of cyclic adenosine monophosphate receptor protein in various virulence traits of Vibrio vulnificus. Microbiol Immunol 2013;57:273-80.

61. Elgaml A, Miyoshi SI. Regulation systems of protease and hemolysin production in Vibrio vulnificus. Microbiol Immunol 2017;61:1-11.

62. Milton DL. Quorum sensing in vibrios: complexity for diversification. Int J Med Microbiol 2006;296:61-71.

63. Lee JH, Rhee JE, Park U, Ju HM, Lee BC, Kim TS, et al. Identification and functional analysis of Vibrio vulnificus SmcR, a novel global regulator. J Microbiol Biotechnol 2007;17:32534.

64. Kim SY, Lee SE, Kim YR, Kim CM, Ryu PY, Choy HE, et al. Regulation of Vibrio vulnificus virulence by the LuxS quorum-sensing system. Mol Microbiol 2003;48:1647-64.

65. Valiente E, Bruhn JB, Nielsen KF, Larsen JL, Roig FJ, Gram L, et al. Vibrio vulnificus produces quorum sensing signals of the AHL-class. FEMS Microbiol Ecol 2009;69:16-26.

66. Wen Y, Kim IH, Kim KS. Iron- and quorum-sensing signals converge on small quorum-regulatory RNAs for coordinated regulation of virulence factors in Vibrio vulnificus. J Biol Chem 2016;291:14213-30.

67. Elgaml A, Higaki K, Miyoshi S. Effects of temperature, growth phase and luxO-disruption on regulation systems of toxin production in Vibrio vulnificus strain L-180, a human clinical isolate. World J Microbiol Biotechnol 2014;30:681-91.

68. Kawase T, Miyoshi S, Sultan Z, Shinoda S. Regulation system for protease production in Vibrio vulnificus. FEMS Microbiol Lett 2004;240:55-9.

69. Kim CM, Shin SH. Modulation of iron-uptake systems by a mutation of luxS encoding an autoinducer-2 synthase in Vibrio vulnificus. Biol Pharm Bull 2011;34:632-7.

70. Kim YR, Lee SE, Kim CM, Kim SY, Shin EK, Shin DH, et al. Characterization and pathogenic significance of Vibrio vulnificus antigens preferentially expressed in septicemic patients. Infect Immun 2003;71:5461-71.

71. Lee SE, Kim SY, Kim CM, Kim MK, Kim YR, Jeong K, et al. The pyrH gene of Vibrio vulnificus is an essential in vivo survival factor. Infect Immun 2007;75:2795-801.

72. Konkel ME, Tilly K. Temperature-regulated expression of bacterial virulence genes. Microbes Infect 2000;2:157-66.

73. Kim CM, Ahn YJ, Kim SJ, Yoon DH, Shin SH. Temperature change induces the expression of vuuA encoding vulnibactin receptor and crp encoding cyclic AMP receptor protein in Vibrio vulnificus. Curr Microbiol 2016;73:54-64.

74. Martínez JL, Delgado-Iribarren A, Baquero F. Mechanisms of iron acquisition and bacterial virulence. FEMS Microbiol Rev 1990;6:45-56.

75. Choi MH, Park RY, Sun HY, Kim CM, Bai YH, Lee SE, et al. Suppression and inactivation of Vibrio vulnificus hemolysin in cirrhotic ascites, a human ex vivo experimental system. FEMS Immunol Med Microbiol 2006;47:226-32.

76. Okujo N, Akiyama T, Miyoshi S, Shinoda S, Yamamoto S. Involvement of vulnibactin and exocellular protease in utilization of transferrin- and lactoferrin-bound iron by Vibrio vulnificus. Microbiol Immunol 1996;40:595-8.

77. Shin SH, Sun HY, Park RY, Kim CM, Kim SY, Rhee JH. Vibrio vulnificus metalloprotease VvpE has no direct effect on the iron-assimilation from human holotransferrin. FEMS Microbiol Lett 2005;247:221-9.

78. Lee HJ, Kim JA, Lee MA, Park SJ, Lee KH. Regulation of haemolysin (VvhA) production by ferric uptake regulator (Fur) in Vibrio vulnificus: repression of vvhA transcription by Fur and proteolysis of VvhA by Fur-repressive exoproteases. Mol Microbiol 2013;88:813-26.

79. Kim CM, Chung YY, Shin SH. Iron differentially regulates gene expression and extracellular secretion of Vibrio vulnificus cytolysin-hemolysin. J Infect Dis 2009;200:582-9.

80. Bang YB, Lee SE, Rhee JH, Choi SH. Evidence that expression of the Vibrio vulnificus hemolysin gene is dependent on cyclic AMP and cyclic AMP receptor protein. J Bacteriol 1999;181:7639-42.

81. Choi HK, Park NY, Kim DI, Chung HJ, Ryu S, Choi SH. Promoter analysis and regulatory characteristics of vvhBA encoding cytolytic hemolysin of Vibrio vulnificus. J Biol Chem 2002;277:47292-9.

82. Jeong HS, Jeong KC, Choi HK, Park KJ, Lee KH, Rhee JH, et al. Differential expression of Vibrio vulnificus elastase gene in a growth phase-dependent manner by two different types of promoters. J Biol Chem 2001;276:13875-80.

83. Jeong HS, Lee MH, Lee KH, Park SJ, Choi SH. SmcR and cyclic AMP receptor protein coactivate Vibrio vulnificus vvpE encoding elastase through the RpoS-dependent promoter in a synergistic manner. J Biol Chem 2003;278:45072-81.

84. Kim CM, Shin SH. Regulation of the Vibrio vulnificus vvpE expression by cyclic AMP-receptor protein and quorum-sensing regulator SmcR. Microb Pathog 2010;49:348-53.

85. Kim CM, Shin SH. Change of Vibrio vulnificus metalloprotease VvpE production by temperature and salinity. J Bacteriol Virol 2011;41:147-56. 
86. Kim CM, Shin SH. SmcR, the quorum-sensing master regulator, is partially involved in temperature/salinity-mediated changes in metalloprotease vvpE expression in Vibrio vulnificus. J Bacteriol Virol 2012;42:29-39.

87. Liu M, Alice AF, Naka H, Crosa JH. The HlyU protein is a positive regulator of rtxA1, a gene responsible for cytotoxicity and virulence in the human pathogen Vibrio vulnificus. Infect Immun 2007;75:3282-9.

88. Shao CP, Lo HR, Lin JH, Hor LI. Regulation of cytotoxicity by quorum-sensing signaling in Vibrio vulnificus is mediated by SmcR, a repressor of hlyU. J Bacteriol 2011;193:2557-65.

89. Elgaml A, Miyoshi S. Role of the histone-like nucleoid structuring protein (H-NS) in the regulation of virulence factor expression and stress response in Vibrio vulnificus. Biocontrol Sci 2015;20:263-74.

90. Childers BM, Klose KE. Regulation of virulence in Vibrio cholerae: the ToxR regulon. Future Microbiol 2007;2:335-44.

91. Lee SE, Shin SH, Kim SY, Kim YR, Shin DH, Chung SS, et al. Vibrio vulnificus has the transmembrane transcription activator ToxRS stimulating the expression of the hemolysin gene vvhA. J Bacteriol 2000;182:3405-15.

92. Kim SP, Kim CM, Shin SH. Cyclic AMP and cyclic AMP-re- ceptor protein modulate the autoinducer-2-mediated quorum sensing system in Vibrio vulnificus. Curr Microbiol 2012;65:701-10.

93. De Lorenzo V, Herrero M, Giovannini F, Neilands JB. Fur (ferric uptake regulation) protein and CAP (catabolite-activator protein) modulate transcription of fur gene in Escherichia coli. Eur J Biochem 1988;173:537-46.

94. Lee HJ, Park KJ, Lee AY, Park SG, Park BC, Lee KH, et al. Regulation of fur expression by RpoS and fur in Vibrio vulnificus. J Bacteriol 2003;185:5891-6.

95. Lee HJ, Bang SH, Lee KH, Park SJ. Positive regulation of fur gene expression via direct interaction of fur in a pathogenic bacterium, Vibrio vulnificus. J Bacteriol 2007;189:2629-36.

96. Kim IH, Wen Y, Son JS, Lee KH, Kim KS. The fur-iron complex modulates expression of the quorum-sensing master regulator, SmcR, to control expression of virulence factors in Vibrio vulnificus. Infect Immun 2013;81:2888-98.

97. Lee HJ, Park SJ, Choi SH, Lee KH. Vibrio vulnificus rpoS expression is repressed by direct binding of cAMP-cAMP receptor protein complex to its two promoter regions. J Biol Chem 2008;283:30438-50. 